\title{
The Angular Momenta, Dipole Moments and Gyromagnetic Ratios of the Neutron and the Muon
}

\author{
Andreas Georgiou \\ School of Physics, Astronomy and Mathematics, University of Hertfordshire, Hatfield, UK \\ Email: a.georgiou@herts.ac.uk \\ Received 18 March 2015; accepted 27 June 2015; published 30 June 2015 \\ Copyright () 2015 by author and Scientific Research Publishing Inc. \\ This work is licensed under the Creative Commons Attribution International License (CC BY). \\ http://creativecommons.org/licenses/by/4.0/ \\ (c) (i) Open Access
}

\section{Abstract}

The dipole moments, angular momenta and gyromagnetic ratios of the electron and the proton were obtained earlier. In this note, we derive the corresponding expressions for the neutron and the muon. This work relies on the results obtained earlier for the angular momenta and dipole moments of rotating spherical bodies.

\section{Keywords}

\section{Angular Momenta, Dipole Moments, Neutron, Muon}

\section{Introduction}

The purpose of this note is to derive analytical formulae for the dipole moments, angular momenta and gyromagnetic ratios of the neutron and the muon. The background to this work is fully explained in reference [1] and a parallel paper on the electron and neutron [2] follows the same methods as presented here.

\section{The Electromagnetic Field Equations}

We shall express the Electromagnetic Field Equations in terms of the 3-vectors representing the electric and magnetic intensities and the corresponding inductions $\boldsymbol{E}, \boldsymbol{H}, \boldsymbol{D}, \boldsymbol{B}$ as follows:

$$
\boldsymbol{E}=E_{a}=F_{a 4} \quad \boldsymbol{D}=D^{a}=-\sqrt{F} F^{a 4} \quad \boldsymbol{H}=H_{a}=\frac{1}{2} \sqrt{F} e_{a k p} F^{k p} \quad \boldsymbol{B}=B^{a}=\frac{1}{2} e^{a k p} F_{k p} .
$$

Here, $e_{a k p}=\sqrt{-\gamma} \varepsilon_{a k p} \quad e^{a k p}=\varepsilon_{a k p} / \sqrt{-\gamma}$ are the covariant and contravariant forms of the completely anti- 
symmetric permutation tensors, $\sqrt{-\gamma}=\sqrt{-g} / \sqrt{F}=F^{-3 / 2} r^{2} \sin \theta$ is the determinant of the spatial metric tensor $\gamma_{a b}=g_{a b}+\gamma_{a} \gamma_{b}$ with $\gamma_{a}=g_{a 4} / \sqrt{F}$, and $\varepsilon_{a k p}$ is the Levi-Civita symbol.

For the details of how these expressions are derived, see [1].

\section{The Neutron}

The mass of the neutron, its classical radius, the square of the classical radius, and the vacuum speed of light, are $m_{n}, a_{n}, a_{n}^{2}$ and $c$.

The following quantities are required:

$$
\begin{array}{lll}
m_{n} \sqrt{G}=4.32663121 \times 10^{-6} \text { esu } & m_{n}=1.674954386 \times 10^{-24} \mathrm{gm} & a_{n}=1.113241576 \times 10^{-12} \mathrm{~cm} \\
m_{n} / a_{n}=1.50457405 \times 10^{-12} & G / c^{2}=7.4242465 \times 10^{-29} & G / c^{4}=8.26058829 \times 10^{-50} \\
\sqrt{G} / c=8.61640674 \times 10^{-15} . & &
\end{array}
$$

Associated with $m_{n}$ there is an electric charge $m_{n} \sqrt{G}$ whose numerical value is given by the first of equations (2) above [1]. If there is an additional charge $q$ then the total electric charge will be $m_{n} \sqrt{G}+q$ esu. We now choose $q$ to be $q=-m_{n} \sqrt{G}$, so that the total charge is zero as required in the case of the neutron. If the total electric charge is zero, the coefficient $F$ of $d t^{2}$ in the Reissner-Nordstrom solution is

$$
F=\left\{1-\frac{2 G m_{n} r / c^{2}}{r^{2}+j^{2} \cos ^{2} \theta}\right\}
$$

where $j$ is the angular momentum per unit mass [3]. On $\theta=\pi / 2, \cos \theta=0$ and so on $r=a$ and $\theta=\pi / 2$ Equation (3) becomes

$$
F=1-\frac{2 G m_{n}}{c^{2} a_{n}}
$$

which is the same as Equation (2) of [3]. In the case of the neutron, it follows from Equation (78) of [1], that for $r=a_{n}$ we have

$$
\lambda^{2}=\frac{2 G m_{n}}{c^{2} a_{n}}+\left[\frac{G m_{n}}{c^{2} a_{n}}\right]^{2}
$$

The term $\left[G m_{n} / a_{n} c^{2}\right]^{2}$ is negligible compared with $2 G m_{n} / c^{2} a_{n}$ and so Equation (5) becomes

$$
\lambda^{2}=\frac{2 G m_{n}}{c^{2} a_{n}}
$$

In accordance with the results of [1], the dipole moment $P_{n}$, total angular momentum $J_{n}$ and gyromagnetic ratio $P_{n} / J_{n}$ are

$$
P_{n}=\frac{3}{2} b^{2} a^{2} \lambda\left(1-\lambda^{2}\right) \frac{c^{2}}{\sqrt{G}} \quad J_{n}=\frac{3}{2} b a^{2} \lambda \frac{c^{3}}{G} \quad \frac{P_{n}}{J_{n}}=b\left(1-\lambda^{2}\right) \frac{\sqrt{G}}{c}
$$

where $\lambda^{2}$ is given by Equation (6).

The values in (2) and Equation (6) give for $\lambda^{2}$ and $\lambda$

$$
\lambda^{2}=2.233980476 \times 10^{-40} \quad \lambda=1.49465062 \times 10^{-20} .
$$

From (6) and the first of (8) we obtain $G m_{n} / c^{2} a_{n}=1.116990238 \times 10^{-20}$ and so $C(a)=1+G m_{n} / a_{n} c^{2}=1+1.116990238 \times 10^{-20}$. Since $1.116990238 \times 10^{-20}$ is negligible compared to 1 , we may write $C(a)=1+G m_{n} / a_{n} c^{2} \approx 1$. From Equations (79) of [1], we obtain

$$
b=1 / C(a)=\frac{1}{1+G m_{n} / a_{n} c^{2}} \approx 1 .
$$

Equations (7) give for $P_{n}$ and $J_{n}$ 


$$
P_{n}=9.662364 \times 10^{-20} \mathrm{erg} / \text { gauss }=9.662364 \times 10^{-23} \mathrm{~J} / \mathrm{T} ; J_{n}=1.12206942 \times 10^{-5} .
$$

It follows that the numerical value of $P_{n} / J_{n}$ is

$$
\frac{P_{n}}{J_{n}}=8.611199831 \times 10^{-15} \text {. }
$$

We note the important fact that this number, is precisely the value of

$$
\sqrt{G} / c \text {. }
$$

\section{The Muon}

The mass $m_{\mu}$ and classical radius $a_{\mu}$ of the muon and its square are

$$
m_{\mu}=1.8219068 \times 10^{-25} \mathrm{~g} \quad a_{\mu}=1.165916 \times 10^{-1} \mathrm{~cm} \quad a_{\mu}^{2}=1.359369446 \times 10^{-2} .
$$

We then obtain

$$
\frac{m_{\mu}}{a_{\mu}}=1.5626344986 \times 10^{-24}
$$

For $\lambda^{2}$ we have

$$
\lambda^{2}=\frac{2 G m_{\mu}}{c^{2} a_{\mu}}-\frac{G q^{2}}{c^{4} a_{\mu}^{2}}
$$

If $q=e$ then $G q^{2} / c^{4} a_{\mu}^{2}$ becomes $G e^{2} / c^{4} a_{\mu}^{2}$. This is negligible compared to $2 G m_{\mu} / c^{2} a_{\mu}$ and so Equation (14) becomes

$$
\lambda^{2}=2 G m_{\mu} / c^{2} a_{\mu}=2.320276742 \times 10^{-47} \quad \lambda=4.8169251 \times 10^{-24} .
$$

Equations (7) will then give

$$
P_{\mu}=10.80672029 \times 10^{-4} \mathrm{erg} / \text { gauss } \quad J_{\mu}=1.254194002 \times 10^{11} \quad P_{\mu} / J_{\mu}=8.616466253 \times 10^{-15} .
$$

This number for the ratio $P_{\mu} / J_{\mu}$ is precisely the value of $\sqrt{G} / c$ and so we have shown that

$$
P_{\mu} / J_{\mu}=\sqrt{G} / c
$$

as in the case of the neutron.

\section{Conclusion}

We have obtained the dipole moments angular momenta and gyromagnetic ratios of the neutron and the muon using the analytical formulae developed in [1]. The values found, are consistent with the expected values of these quantities. In particular, the ratio $P / J$ has the value $\sqrt{G} / c=8.61640674 \times 10^{-15}$ in both the case of the neutron and in the case of the muon. We also note that $P / J$ has the same value as in the case of the electron and the proton [2] and as in the case of other rotating spherical bodies [1]. It is indeed remarkable that the ratio $P / J$ is first developed by us, to deal with rotating spherical bodies of arbitrary masses and radii and we apply it to the case of rotating stars. We now find that it is also valid for elementary particles, which in the classical approximation are assumed to be spherical.

\section{References}

[1] Georgiou, A. (2012) Journal of Modern Physics, 3, 1301-1310. http://dx.doi.org/10.4236/jmp.2012.329168

[2] Georgiou, A. (2014) Journal of Modern Physics, 5, 1254-1257. http://dx.doi.org/10.4236/jmp.2014.514125

[3] Blinder, S.M. (2003) Dirac’s Equation via General Relativity. Electromagnetic Phenomena, PACS No. 03.50.De; 14.60.C. http://www.emph.com.ua/9/pdf/blinder.pdf 